\title{
Modeling of the Crystal Structure \\ of Platinum Metal's Complex Compounds \\ by Using Parallel Computing Based \\ on Genetic Algorithms and X-ray Diffraction Data
}

\author{
Alexander N. Zaloga ${ }^{a}$, Peter S. Dubinin ${ }^{a}$, \\ Sergey D. Kirik ${ }^{\mathrm{a}}$, Ruslan F. Mulagaleev ${ }^{\mathrm{b}}$, \\ Leonid A. Solovyev and Igor S. Yakimov*a \\ ${ }^{a}$ Siberian Federal University \\ 79 Svobodny, Krasnoyarsk, 660041, Russia \\ ${ }^{b} J S C$ «Krasnoyarsk Nonferrous Metals Plant» \\ 1 Transport fares, Krasnoyarsk, 660027, Russia \\ 'Institute of Chemistry and Chemical Technology SB RAS \\ 50/24 Akademgorodok, Krasnoyarsk, 660036, Russia
}

Received 09.04.2015, received in revised form 27.08.2015, accepted 03.02.2016

Crystal structure models of complex compounds [Pd(CH3NH2)4][PdBr4] (sp. gr. P4/mnc (128), $a=10.6866(7) A, c=6.7262(3) \AA, V=768.16(10) \AA 3$ ) and $[P t(N H 3) 5 C l] B r 3$ (sp. gr. I41/a (88), $a=17.2587(5) \AA ; c=15.1164(3) \AA, V=4502,61(10) A 3)$ has been determined by using the developed multipopulational parallel genetic algorithm (MPGA) and x-ray powder diffraction data. This paper presents the methodology and results of the structural analysis of these compounds obtained by application of the MPGA.

Keywords: crystal structure analysis, X-ray powder diffraction, genetics algorithm, full-profile analysis, Rietveld method.

Citation: Zaloga A.N., Dubinin P.S., Kirik, S.D., Mulagaleev, R.F., Solovyev, L.A., Yakimov I.S. Modeling of the crystal structure of platinum metal's complex compounds by using parallel computing based on genetic algorithms and $\mathrm{x}$-ray diffraction data, J. Sib. Fed. Univ. Eng. technol., 2016, 9(1), 105-116. DOI: 10.17516/1999-494X-2016-9-1-105-116.

(C) Siberian Federal University. All rights reserved

* Corresponding author E-mail address: i-s-yakimov@yandex.ru 


\title{
Моделирование кристаллической структуры \\ комплексных соединений платиновых металлов \\ с помощью параллельных вычислений
}

на основе генетических алгоритмов

и данных рентгеновской дифракции

\author{
А.Н. Залога ${ }^{\text {a }, ~ П . С . ~ Д у б и н и н ~}{ }^{a}$, С.Д. Кирик ${ }^{a}$, \\ Р.Ф. Мулагалеев ${ }^{\sigma}$, Л.А. Соловьев ${ }^{\text {в }}$, И.С. Якимов ${ }^{\text {a }}$ \\ ${ }^{a}$ Сибирский федеральнылй университет \\ Россия, 660041, Красноярск, Свободный, 79 \\ ${ }^{6} О А О$ «Красноярский завод ияветных металлов» \\ Россия, 660027, Красноярск, Транспортный проезд, 1 \\ ${ }^{8}$ Институт химии и химической технологии СО РАН \\ Россия, 660036, Красноярск, Академгородок, 50/24
}

Модели кристаллической структуры комплексных соединений $\left[\mathrm{Pd}\left(\mathrm{CH}_{3} \mathrm{NH}_{2}\right)_{4}\right]\left[\mathrm{PdBr}_{4}\right]$ (np.гp. P4/mnc (128), $\left.a=10.6866(7) \AA, c=6.7262(3) \AA, V=768.16(10) A^{3}\right) u\left[P t\left(N_{3}\right)_{5} C l_{1} B r_{3}(n p . ~ z p . ~ I 41 / a(88)\right.$, параметры ячейки $\left.a=17.2587(5) A ; c=15.1164(3) A, V=4502,61(10) \AA^{3}\right)$ определены с помощью разработанного мультипопуляцчионного параллельного генетического алгоритма (МПГА) и данных рентгеновской порошковой дифракции. Обсуждаются методика и результать структурного анализа этих соединений по МПГА.

Ключевые слова: кристаллическая структура, рентгеновская порошковая дифракиия, эволюционные генетические алгоритмы, полнопрофильный анализ, метод Ритвельда.

\section{Введение}

Информация об атомной кристаллической структуре веществ содержит координаты атомов в элементарной кристаллической ячейке и параметры их тепловых колебаний, накапливается в структурных базах данных $[1,2]$ и используется для объяснения и прогнозирования физических и химических свойств веществ и материалов. Для веществ, получаемых в поликристаллической форме, основным методом изучения структуры служит рентгеновская порошковая дифракция. Исследование структуры включает определение приближенной модели атомной кристаллической структуры и ее оптимизацию. Оптимизация структурной модели осуществляется с помощью метода Ритвельда [3], основанного на расчете дифрактограмм из атомной кристаллической структуры и минимизации относительной невязки между расчетной и экспериментальной дифрактограммами (профильного R-фактора) по нелинейному MHК.

Основной проблемой дифракционного структурного анализа является поиск модели кристаллической структуры. С ростом мощности компьютеров эффективными для этой цели стали методы глобальной оптимизации структуры в прямом пространстве [4, 5], основанные, подобно методу Ритвельда, на моделировании экспериментальных дифрактограмм, в частности генетические алгоритмы (ГА) [6]. ГА имитируют биологическую эволюцию, т.е. осуществляют поэтапную стохастическую глобальную оптимизацию сразу целого множества (популяции) случайных структурных моделей путем их парной рекомбинации, случайных изменений атом- 
ных координат (мутаций) и селекции по R-фактору лучших моделей в новые поколения популяции с постепенным накоплением в них истинных позиций атомов в структуре. Параллельная эволюционная оптимизация - важное преимущество ГА. Механизм сходимости поиска структуры по ГА изучен нами в [7]. Основным недостатком является частая преждевременная стагнация процесса поиска в локальных минимумах R-фактора - до сходимости одной из структурных моделей к истинной структуре вещества. В [8] нами предложен мультипопуляционный параллельный генетический алгоритм (МПГА), снижающий вероятность стагнации за счет обмена структурными моделями между независимыми популяциями.

Исходными данными для определения модели структуры служат химическая формула, параметры кристаллической ячейки, пространственная группа симметрии и полнопрофильная порошковая дифрактограмма вещества. Для учета кристаллохимических особенностей структуры возможно задание априори известных структурных фрагментов и «мягких» ограничений на длины межатомных расстояний. Предварительно по методу Ле Бэйла [9] выполняется моделирование профиля дифрактограммы с подгонкой профильных параметров, таких как форма и ширина дифракционных рефлексов и т.п., которые затем фиксируются. Достигнутое при этом значение профильного R-фактора служит целевым при дальнейшем поиске структуры по МПГА. В [10] обсуждаются несколько вариантов реализации МПГА: с битовым и действительным кодированием атомных координат, с промежуточной локальной оптимизацией структурных моделей по встроенным программам полнопрофильного анализа DDM [11] и Ритвельда из [12], для многоядерных ПК и вычислительного кластера СФУ, а также результаты исследования их сходимости при поиске известных кристаллических структур ряда веществ. В данной статье описываются методика и результаты определения с помощью МПГА ранее неизвестной кристаллической структуры комплексных соединений платиновых металлов.

\section{Объекты и методы исследования}

\section{Объекты исследования}

Объектами исследования являются атомные кристаллические структуры комплексных соединений $\left[\mathrm{Pd}\left(\mathrm{CH}_{3} \mathrm{NH}_{2}\right)_{4}\right]\left[\mathrm{PdBr}_{4}\right]$ и $\left[\mathrm{Pt}\left(\mathrm{NH}_{3}\right)_{5} \mathrm{Cl}\right] \mathrm{Br}_{3}$, синтезированные из стандартных химических реактивов чистотой не ниже “х.ч. - ч.д.а." Синтез $\left[\mathrm{Pd}\left(\mathrm{CH}_{3} \mathrm{NH}_{2}\right)_{4}\right]\left[\mathrm{PdBr}_{4}\right]$ был выполнен следующим образом. Концентрированный раствор $\mathrm{H}_{2} \mathrm{PdBr}_{4}$, полученный растворением палладиевой черни в смеси бромистоводородной кислоты и брома и упаренный до начала кристаллизации $\mathrm{PdBr}_{2}$, разбавили водой в 4 раза и охладили до $0{ }^{\circ} \mathrm{C}$. В полученный раствор добавили $10-15 \%$-й водный раствор метиламина $\left(\mathrm{CH}_{3} \mathrm{NH}_{2}\right)$, также охлаждённый до $0{ }^{\circ} \mathrm{C}$, до достижения $\mathrm{pH}$ раствора 6-8. В результате образовался мелкокристаллический розовый осадок. Синтез $\left[\mathrm{Pt}\left(\mathrm{NH}_{3}\right)_{5} \mathrm{Cl}\right] \mathrm{Br}_{3}$ выполнен следующим образом. Комплексное соединение $\left[\mathrm{Pt}\left(\mathrm{NH}_{3}\right)_{5} \mathrm{Cl}_{1} \mathrm{Cl}_{3}{ }^{*} \mathrm{H}_{2} \mathrm{O}\right.$, полученное в соответствии с [13], растворили в минимальном количестве воды при $30-40{ }^{\circ} \mathrm{C}$ и добавили концентрированный раствор бромида калия в мольном соотношении $\mathrm{Pt}: \mathrm{Br}=1$ : (3-4). Образовавшиеся осадки этих соединений отфильтровали, промыли водой и затем этанолом и сушили на фильтре. Дифрактограммы получены с использованием СuK $\alpha$ излучения на дифрактометре X'Pert PRO с детектором PIXcel (PANalytical), снабженным графитовым монохроматором. Условия съемки: диапазон от 5 до $90^{\circ}$ по шкале $2 \theta$, шагом $0,026^{\circ}, \Delta \mathrm{t}-50 \mathrm{c}$. В результате индицирования дифрактограмм и определения пространственной группы симметрии при помощи программы ЕХРО [14] определены

$$
-107 \text { - }
$$


следующие кристаллографические характеристики этих соединений: $\left[\mathrm{Pd}\left(\mathrm{CH}_{3} \mathrm{NH}_{2}\right)_{4}\right]\left[\mathrm{PdBr}_{4}\right]-$ пр. гр. $P 4 / m n c$, параметры ячейки $a=10.6866(7) \AA, c=6.7262(3) \AA, V=768.16(10) \AA^{3} ;\left[\mathrm{Pt}\left(\mathrm{NH}_{3}\right)_{5} \mathrm{Cl}\right] \mathrm{Br}_{3}-$ пр. гр. I41/a, параметры ячейки а=17.2587(5) $\AA$; $=15.1164(3) \AA ⿻$, $V=4502,61(10) \AA^{3}$. Соответствие всех позиций дифракционных рефлексов расчетным значениям, вычисленным по параметрам кристаллической решетки, подтвердило чистоту синтеза.

\section{Методы исследования}

Определение кристаллической структуры выполнялось с помощью МПГА на компьютере с процессором Intel i73770, имеющем 8 вычислительных ядер: 7 для процессов эволюционного поиска структуры в независимых популяциях и одно в качестве управляющего для регулярного накопления лучших структурных моделей из популяций и межпопуляционного обмена [8]. Был выбран вариант МПГА [10] с вещественным кодированием атомных координат и периодической локальной оптимизацией лучших структурных моделей по программе МНК метода Ритвельда. Процесс эволюционного поиска структуры после запуска МПГА выполняется автоматически, контроль осуществляется визуально по графикам сходимости, распределения атомов популяций в кристаллической ячейке и соответствия экспериментальной дифрактограммы с расчетными для лучших структурных моделей, получаемых на данном этапе эволюции. Для работы МПГА требуется предварительная настройка параметров поиска, от качества которой зависит вероятность сходимости структурных моделей к истинной структуре. Настройка была выполнена путем эмпирического выбора параметров в процессах поиска известных кристаллических структур соединений $\left[\mathrm{Pd}\left(\mathrm{NH}_{3}\right)_{4}\right]\left(\mathrm{C}_{2} \mathrm{O}_{4}\right)$ и $\left[\mathrm{Pt}\left(\mathrm{NH}_{3}\right)_{2}\left(\mathrm{C}_{2} \mathrm{O}_{4}\right)\right]$, близких по составу к исследуемым. Выбранные настройки приведены в табл. 1.

После сходимости МПГА к значениям R-фактора, близким к целевому значению, выполняется кристаллохимическая верификация найденной структуры, включающая оценку коррект-

Таблица 1. Настройки параметров МПГА, выбранные для поиска структур

\begin{tabular}{|l|c|}
\hline \multicolumn{1}{|c|}{ Параметр } & 3начение \\
\hline Размер популяций (количество структурных моделей) & 300 \\
\hline Регулярность запуска локального поиска по МНК, поколений & 5 \\
\hline Циклов МНК при запуске локального поиска & 3 \\
\hline $\begin{array}{l}\text { Количество структурных моделей, которые подвергались локальному поиску при } \\
\text { каждом запуске МНК }\end{array}$ & 9 \\
\hline $\begin{array}{l}\text { Регулярность отправки структурных моделей с управляющего ядра на рабочие, } \\
\text { поколений }\end{array}$ & 2 \\
\hline Количество отправляемых структурных моделей & 3 \\
\hline $\begin{array}{l}\text { Количество поколений для локального элитизма (временного сохранения в популяции } \\
\text { лучшей структурной модели) }\end{array}$ & 3 \\
\hline $\begin{array}{l}\text { Размер турнира (количество структурных моделей для турнирного отбора при } \\
\text { селекции) }\end{array}$ & 0,75 \\
\hline $\begin{array}{l}\text { Коэффициент скрещивания (доля структурных моделей, участвующих в вероятностной } \\
\text { парной рекомбинации их атомов) }\end{array}$ & 1,5 \\
\hline Коэффициент мутации атомных координат структурной модели & 5 \\
\hline
\end{tabular}


ности межатомных расстояний, валентных углов и т.П., расстановку атомов водорода, уточнение дифрактограммы по методу Ритвельда, а также контроль корректности структуры по on-line-программе CheckCIF [15] для ее опубликования.

\section{Результаты и их обсуждение}

Определение структуры соединения $\left[\mathrm{Pt}\left(\mathrm{NH}_{3}\right)_{5} \mathrm{Cl}\right] \mathrm{Br}_{3}$

В результате моделирования по методу Ле Бэйла определены профильные параметры дифрактограммы и целевое значение профильного R-фактора, равное 5,49 \% отн. По данным статистики распределения межатомных расстояний в структурах разных соединений аналогичного состава, рассчитанной по программе Diamond [16], были наложены ограничения на длины межатомных расстояний, приведенные в табл. 2. Аналогично установлены ограничения на минимальные межатомные расстояния для других пар атомов.

При поиске структуры по МПГА искомыми параметрами являлось расположение в ячейке трех атомов $\mathrm{Br}$ и априори известного структурного фрагмента $\mathrm{PtN}_{5} \mathrm{Cl}$. На этом этапе атомы водорода не учитывались, а параметры изотропных тепловых колебаний атомов были взяты из структур близкого состава. Перечень параметров приведен в табл. 3.

На рис. 1 представлены графики сходимости МПГА при поиске этой структуры.

По оси абсцисс указаны номера поколений эволюции (нулевой номер - начальная случайная генерация всех популяций), по оси ординат - соответствующие значения функции пригодности (R-фактор плюс штраф за нарушение структурных ограничений) лучшей структурной модели из числа накопленных на управляющем ядре. Красная линия показывает значение пригодности лучшей из найденных структурных моделей. Чёрная линия - значение лучшей пригодности, полученной после уточнения лучшей структурной модели по МНК (запаздывает, если установлен режим выполнения МНК раз в несколько поколений). Фиолетовая линия -

Таблица 2. Значения межатомных расстояний для атомов, координированных к $\mathrm{Pt}$

\begin{tabular}{|l|c|c|c|c|}
\hline \multicolumn{1}{|c|}{ Межатомные расстояния, $\AA$} & Pt-Cl & Pt-N & Pt-Br & Pt-Pt \\
\hline Минимально допустимое расстояние & 2,15 & 1,8 & 3,95 & 4 \\
\hline Типичное расстояние для химического соединения & 2,32 & 2,15 & - & \\
\hline $\begin{array}{l}\text { Диапазон возможного отклонения от типичного межатомного } \\
\text { расстояния }\end{array}$ & $\pm 0,05$ & $\pm 0,08$ & - & \\
\hline
\end{tabular}

Таблица 3. Степени свободы структурных параметров $\left[\mathrm{Pt}\left(\mathrm{NH}_{3}\right)_{5} \mathrm{Cl}\right] \mathrm{Br}_{3}$ в поиске по МПГА

\begin{tabular}{|l|c|}
\hline \multicolumn{1}{|c|}{ Характеристика структуры } & Кол-во параметров \\
\hline Координаты атома $\mathrm{Br} 1$ & 3 \\
\hline Координаты атома $\mathrm{Br} 2$ & 3 \\
\hline Координаты атома $\mathrm{Br3}$ & 3 \\
\hline Координаты и углы, определяющего ориентацию фрагмента $\mathrm{PtN}_{5} \mathrm{Cl}$ & 7 \\
\hline Общее число степеней свободы структуры & 16 \\
\hline
\end{tabular}




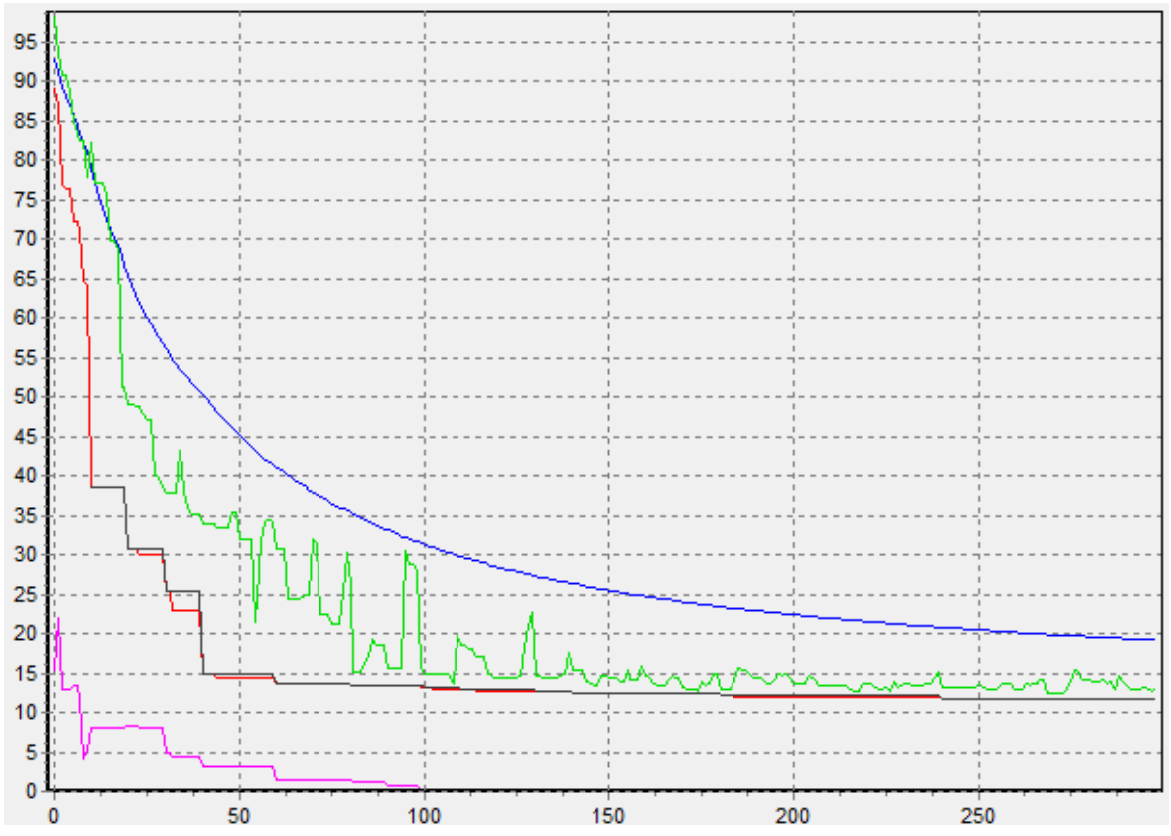

Рис. 1. График сходимости при поиске структуры $\left[\mathrm{Pt}\left(\mathrm{NH}_{3}\right)_{5} \mathrm{Cl}\right] \mathrm{Br}_{3}$ по МПГА; по оси $\mathrm{X}$ - номер поколения; по оси $\mathrm{Y}$ - значение функции пригодности; пригодность лучшей структурной модели - красная линия, величина штрафов - фиолетовая

вклад штрафов в значениях функции пригодности. Синяя - среднее значение пригодности структурных решений, накопленных на управляющем ядре. Зелёная линия - худшее из лучших решений структуры на рабочих ядрах. Совокупность графиков позволяет анализировать и контролировать процесс сходимости.

На рис. 1 видно, что уже к 40-му поколению эволюции достигнуто значение пригодности $15 \%$ и структура в основном сформировалась. Однако она еще недостаточно корректна, т.к. часть межатомных расстояний в ней выходит за ограничения (уровень штрафов около $3 \%$ ). К сотому поколению штрафы исчезают и все межатомные расстояния становятся корректными, а пригодность совпадает со значениями R-фактора. Далее R-фактор постепенно снижается с 13,5 до $12 \%$, в основном за счет уточнения ориентации фрагмента $\mathrm{PtN}_{5} \mathrm{Cl}$, и к 240 -му поколению процесс сходимости завершается. При этом лучшие структурные модели постепенно распространяются по популяциям на рабочих ядрах (минимизируется зелёная линия). Следует отметить, что практически все резкие спады пригодности происходят за счет локальных оптимизаций структурных моделей по МНК (красные линии на них совпадают с черными).

Данная модель структуры уточнена по программе полнопрофильного анализа DDM вместе с параметрами тепловых колебаний, и получен R-фактор 6,78 \%, который был выше целевого на $1,3 \%$. Очевидно, разница обусловлена отсутствием легких атомов водорода в данной структурной модели, которые не могут быть локализованы на этапе поиска модели. На рис. 2 приведено сравнение экспериментальной дифрактограммы с полученной в результате финального уточнения. Координаты неводородных атомов структуры $\left[\mathrm{Pt}\left(\mathrm{NH}_{3}\right)_{5} \mathrm{Cl}\right] \mathrm{Br}_{3}$, их изотропные тепловые параметры и коэффициенты заполнения кристаллографических позиций приведены в табл. 4. 


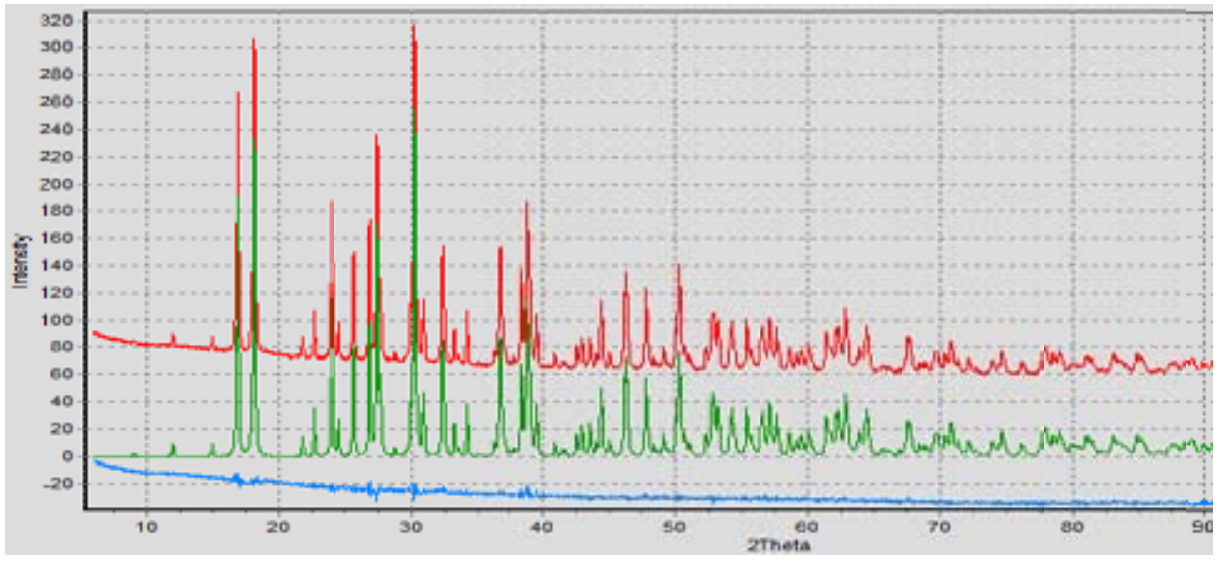

Рис. 2. График сравнения экспериментальной дифрактограммы $\left[\mathrm{Pt}\left(\mathrm{NH}_{3}\right)_{5} \mathrm{Cl}\right] \mathrm{Br}_{3}$ с уточнением по DDM; красная линия обозначает экспериментальную дифрактограмму, зелёная - рассчитанную, синяя - разность между ними.

Таблица 4. Модель кристаллической структуры соединения $\left[\mathrm{Pt}\left(\mathrm{NH}_{3}\right)_{5} \mathrm{Cl}\right] \mathrm{Br}_{3}$

\begin{tabular}{|c|c|c|c|c|c|}
\hline Atom & $\mathrm{X}$ & $\mathrm{Y}$ & $\mathrm{Z}$ & Biso & $\mathrm{N}$ \\
\hline $\mathrm{Pt}$ & $0.2460(1)$ & $0.2685(1)$ & $0.5029(2)$ & $1.638(12)$ & 16 \\
\hline $\mathrm{Br} 1$ & $0.1240(2)$ & $0.2820(3)$ & $0.2311(4)$ & $3.772(17)$ & 16 \\
\hline $\mathrm{Br} 2$ & $0.2842(2)$ & $0.5282(2)$ & $0.4855(3)$ & $3.231(17)$ & 16 \\
\hline $\mathrm{Br} 3$ & $0.1504(3)$ & $0.3075(3)$ & $0.7671(5)$ & $6.844(26)$ & 16 \\
\hline $\mathrm{C} 1$ & $0.1280(6)$ & $0.3273(4)$ & $0.4925(10)$ & $2.236(32)$ & 16 \\
\hline $\mathrm{N} 1$ & $0.3517(13)$ & $0.2154(13)$ & $0.5133(23)$ & $0.95(34)$ & 16 \\
\hline $\mathrm{N} 2$ & $0.2208(19)$ & $0.1944(14)$ & $0.4062(17)$ & $0.95(34)$ & 16 \\
\hline $\mathrm{N} 3$ & $0.2779(19)$ & $0.3500(13)$ & $0.5967(16)$ & $0.95(34)$ & 16 \\
\hline $\mathrm{N} 4$ & $0.2083(18)$ & $0.1939(14)$ & $0.5979(14)$ & $0.95(34)$ & 16 \\
\hline $\mathrm{N} 5$ & $0.2851(15)$ & $0.3418(13)$ & $0.4111(15)$ & $0.95(34)$ & 16 \\
\hline
\end{tabular}

Кристаллическая структура построена из комплексных катионов $\left[\mathrm{Pt}\left(\mathrm{NH}_{3}\right)_{5} \mathrm{Cl}\right]^{3+}$ и трех кристаллографически независимых анионов $\mathrm{Br}-$. Комплексный катион из-за присутствия в нем атома хлора имеет вид неправильного октаэдра с длинами связей: Pt-Cl4 = 2,291

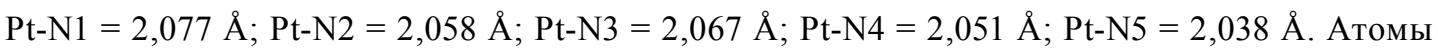
$\mathrm{N} 1$ и Cl4 лежат в вершинах октаэдра, остальные четыре азота в его основании. Углы N-Pt-N в основании октаэдра отклоняются от прямых и меняются от $86,9^{\circ}$ до $93,2^{\circ}$. Угол между атомами в вершинах октаэдра N1-Pt-Cl4 составил $176^{\circ}$. Все атомы располагаются в общих позициях.

\section{Определение структуры соединения $\left[\mathrm{Pd}\left(\mathrm{CH}_{3} \mathrm{NH}_{2}\right)_{4}\right]\left[\mathrm{PdBr}_{4}\right]$}

В результате моделирования по методу Ле Бэйла определены профильные параметры дифрактограммы и целевое значение R-фактора, равное 6,88 \% отн. По данным статистики распределения межатомных расстояний введены ограничения, приведенные на рис. 3. 


\begin{tabular}{|c|c|c|}
\hline Power 1 & Power2 & Min. dist. \\
\hline Pd & Pd & 3 \\
\hline Pd & $\mathrm{N}$ & 1.85 \\
\hline Pd & C & 3 \\
\hline Pd & $\mathrm{Br}$ & 2.2 \\
\hline $\mathrm{N}$ & $N$ & 2.5 \\
\hline$N$ & C & 1.4 \\
\hline $\mathrm{N}$ & $\mathrm{Br}$ & 3.5 \\
\hline C & c & 3 \\
\hline C & $\mathrm{Br}$ & 3.5 \\
\hline $\mathrm{Br}$ & $\mathrm{Br}$ & 3 \\
\hline
\end{tabular}

Рис. 3. Задание минимальных межатомных расстояний в $\left[\mathrm{Pd}\left(\mathrm{CH}_{3} \mathrm{NH}_{2}\right)_{4}\right]\left[\mathrm{PdBr}_{4}\right]$

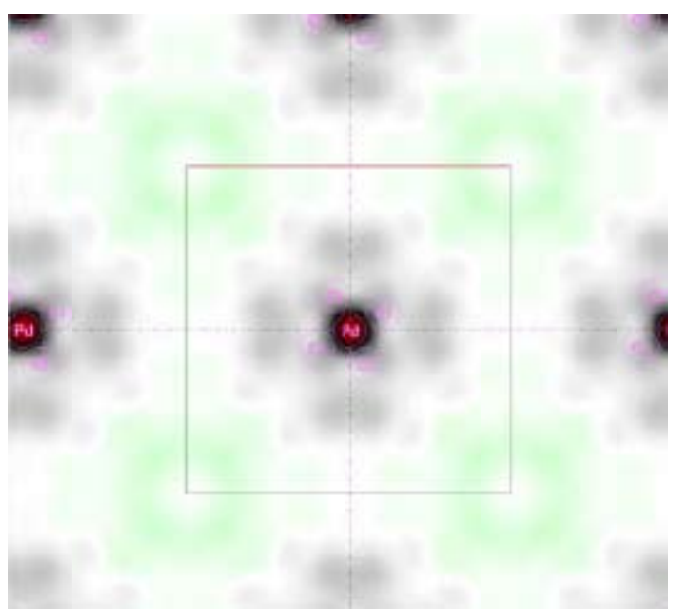

Рис. 4. Карта электронной плотности для $\left[\mathrm{Pd}\left(\mathrm{CH}_{3} \mathrm{NH}_{2}\right)_{4}\right]\left[\mathrm{PdBr}_{4}\right]$, рассчитанная в программе DDM в плоскости $a b$

При анализе карт электронной плотности, в частности, приведенной на рис. 4, было сделано предположение, что тяжелые атомы палладия лежат на грани, перпендикулярной к оси $c$, причём один из них в начале координат, другой - в центре грани, и лёгкие неводородные атомы также должны быть расположены на этой грани.

Перечень структурных параметров для поиска по МПГА приведен в табл. 5.

На рис. 5 представлены графики сходимости МПГА при поиске этой структуры. Мы видим, что уже к 10 -му поколению эволюции достигнуто значение пригодности $13 \%$ и структура в основном сформировалась. Далее происходит уточнение позиций атомов С и N, и к 23-му поколению процесс сходимости практически завершается.

Полученная модель структуры была уточнена по программе DDM, проведено совместное уточнение всех профильных и структурных параметров, а также параметра текстуры для ее эллипсоидной модели (в соответствии с сингонией соединения). Всего было уточнено 19 параметров (10 профильных, 3 параметра текстуры и 6 координат атомов), в итоге был получен 
Таблица 5. Степени свободы структурных параметров $\left[\mathrm{Pd}\left(\mathrm{CH}_{3} \mathrm{NH}_{2}\right)_{4}\right]\left[\mathrm{PdBr}_{4}\right]$ при поиске структуры по МПГА

\begin{tabular}{|l|c|}
\hline \multicolumn{1}{|c|}{ Характеристика структуры } & Кол-во параметров \\
\hline Координаты Х и Y атома C1 & 2 \\
\hline Координаты X и Y атома N1 & 2 \\
\hline Координаты X и Y атома Br1 & 2 \\
\hline Общее число степеней свободы структуры & 6 \\
\hline
\end{tabular}

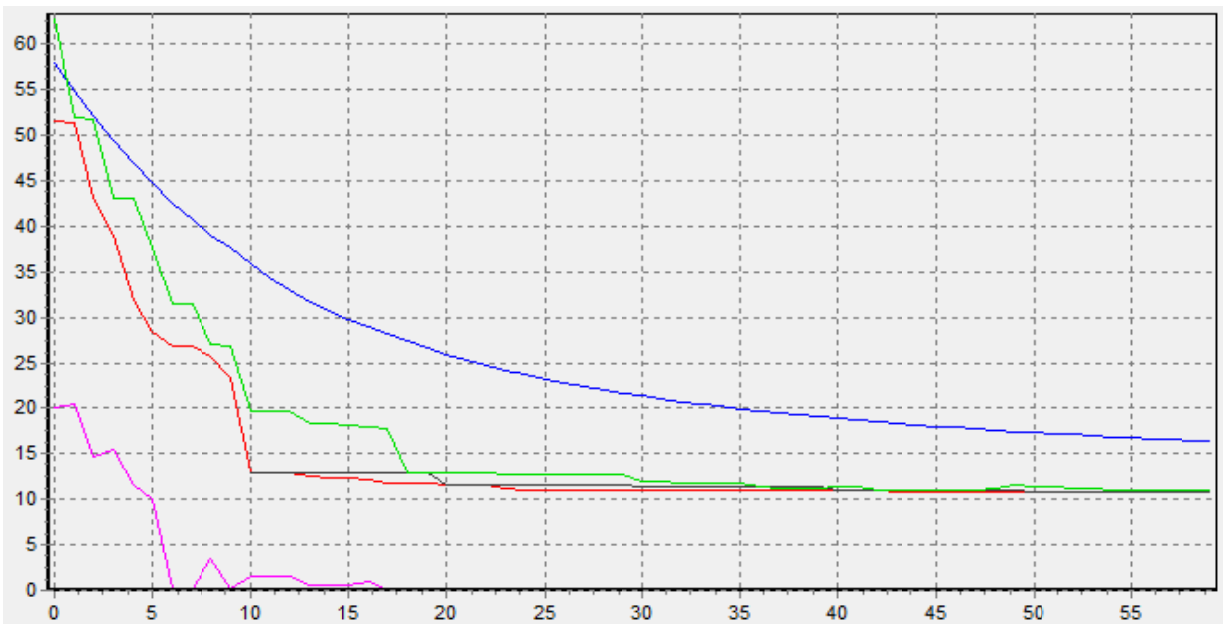

Рис. 5. График сходимости при поиске структуры $\left[\mathrm{Pd}\left(\mathrm{CH}_{3} \mathrm{NH}_{2}\right)_{4}\right]\left[\mathrm{PdBr}_{4}\right]$ по МПГА; по оси X - номер поколения; по оси Y - значение функции пригодности; пригодность лучшей структурной модели - красная линия, величина штрафов - фиолетовая

R-фактор 7,49 \%. Легкие атомы водорода не локализуются на этапе поиска модели. На рис. 6 приведено сравнение экспериментальной дифрактограммы с полученной в результате финального уточнения.

Найденные по МПГА и уточненные по DDM структурные данные (координаты атомов $x, y, z$ и коэффициенты заполнения атомных позиций $N$ ) для независимой части ячейки $\left[\mathrm{Pd}\left(\mathrm{CH}_{3} \mathrm{NH}_{2}\right)_{4}\right]\left[\mathrm{PdBr}_{4}\right]$ приведены в табл. 6.

Найденная кристаллическая структура содержит две плоские квадратные части: $\left[\mathrm{Pd}\left(\mathrm{CH}_{3} \mathrm{NH}_{2}\right)_{4}\right]$ и $\left[\mathrm{PdBr}_{4}\right]$, которые располагаются параллельно друг относительно друга. Группа метиламин координируется к палладию через азот с расстоянием около 2,0542(1) А̊ (угол $\left.P d-N-C 109,798(2)^{\circ}\right)$. Расстояние $N$ - $C$ составляет 1,4561(1) $\AA$; $P d-B r$, соответственно 2,3894(1) $\AA$. Локализованы все атомы водорода. Длина связи $C$...H меняется в диапазоне от $0,9614 \AA$ до $0,9636 \AA$, углы в группе $C H_{3} 111,017^{\circ}$ и $109,936^{\circ}$. Полученные длины связи хорошо соответствуют межатомным расстояниям в структурах комплексных соединений близкого состава (табл. 7).

Соответствие длин межатомных связей, в том числе для водородных атомов, и низкое значение профильного R-фактора свидетельствуют об адекватности найденной кристалли- 


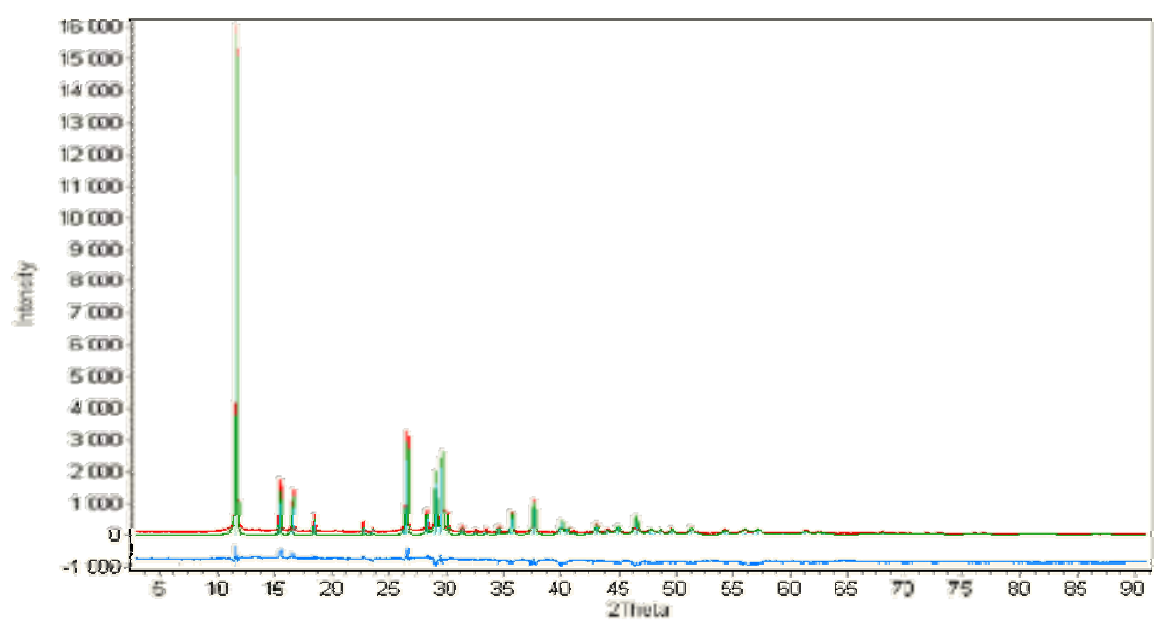

Рис. 6. Экспериментальная и расчетная дифрактограммы $\left[\mathrm{Pd}\left(\mathrm{CH}_{3} \mathrm{NH}_{2}\right)_{4}\right]\left[\mathrm{PdBr}_{4}\right]$ после уточнения по DDM, внизу их разница; $R_{w p}=7,49 \%$

Таблица 6. Модель кристаллической структуры соединения $\left[\mathrm{Pd}\left(\mathrm{CH}_{3} \mathrm{NH}_{2}\right)_{4}\right]\left[\mathrm{PdBr}{ }_{4}\right]$

\begin{tabular}{|c|c|c|c|c|c|}
\hline Атом & $\mathrm{x}$ & $\mathrm{y}$ & $\mathrm{z}$ & Uiso & Occup. \\
\hline $\mathrm{Pd} 1$ & 0 & 0 & 0 & $0.036(6)$ & 2 \\
\hline $\mathrm{Pd} 2$ & 0.5 & 0.5 & 0 & $0.032(8)$ & 2 \\
\hline $\mathrm{N}$ & $0.152(7)$ & $0.117(8)$ & 0 & 0.02 & 8 \\
\hline $\mathrm{C}$ & $0.267(12)$ & $0.044(8)$ & 0 & 0.02 & 8 \\
\hline $\mathrm{Br}$ & $0.2997(8)$ & $0.4007(9)$ & 0 & $0.0273(33)$ & 8 \\
\hline
\end{tabular}

Таблица 7. Сравнение длин связей в $\left[\mathrm{Pd}\left(\mathrm{CH}_{3} \mathrm{NH}_{2}\right)_{4}\right]\left[\mathrm{PdBr}_{4}\right]$ с их длинами в других структурах, взятых из базы структурных данных ICSD, А̊

\begin{tabular}{|c|c|c|c|c|c|}
\hline Формула\№ карточки ICSD & $\mathrm{Pd}-\mathrm{Br}$ & $\mathrm{Pd}-\mathrm{N}$ & $\mathrm{N}-\mathrm{C}$ & $\mathrm{C}-\mathrm{H}$ & $\mathrm{N}-\mathrm{H}$ \\
\hline$\left[\mathrm{Pd}\left(\mathrm{CH}_{3} \mathrm{NH}_{2}\right)_{4}\right]\left[\mathrm{PdBr}_{4}\right]$ & 2,389 & 2,054 & 1,456 & $0,961 \ldots 0,963$ & 0,865 \\
\hline$\left[\mathrm{Cr}\left(\mathrm{NH}_{3}\right)_{5} \mathrm{Cl}\right]\left[\mathrm{PdBr}_{4}\right] /$ №158877 & 2,413 & & & & \\
\hline$\left(\mathrm{NH}_{4}\right)_{2}\left(\mathrm{PdBr}_{4}\right)$ / №202799 & 2,437 & & & & \\
\hline$\left[\left(\mathrm{CH}_{3}\right)_{2} \mathrm{NH}_{2}\right]_{2}\left[\mathrm{PdBr}_{4}\right]$ / №110463 & 2,434 & & 1,431 & & \\
\hline $\mathrm{Pd}\left(\mathrm{NH}_{3}\right)_{2} \mathrm{Br}_{2} /$ №280377 & 2,$246 ; 2,440$ & 2,037 & & & \\
\hline$\left(\mathrm{NH}_{3}\right)_{4} \mathrm{Pd}\left(\mathrm{N}_{3}\right)_{4} \mathrm{Pd} /$ №410384 & & 2,056 & & & 0,912 \\
\hline$\left(\mathrm{NH}_{2}\left(\mathrm{CH}_{3}\right)\right)\left(\mathrm{BH}_{3}\right) /$ №249797 & & & 1,475 & $0,959 \ldots 0,969$ & 0,882 \\
\hline $\mathrm{N}\left(\mathrm{CH}_{3}\right)_{4} \mathrm{CuZn}(\mathrm{CN})_{4} /$ №249948 & & & & 0,979 & \\
\hline
\end{tabular}


ческой структуры соединения $\left[\mathrm{Pd}\left(\mathrm{CH}_{3} \mathrm{NH}_{2}\right)_{4}\right]\left[\mathrm{PdBr}_{4}\right]$. Контроль этой структуры по программе CheckCIF также подтвердил ее корректность.

\section{Заключение}

Генетические алгоритмы обеспечивают ab initio поиск атомной кристаллической структуры химических соединений по порошковой дифрактограмме, хорошо автоматизируются и имеют существенный, но пока практически неиспользуемый потенциал развития за счет организации параллельных вычислений на многоядерных ПК и суперкомпьютерных кластерах. Вариант данного подхода реализован нами в МПГА и успешно апробирован при поиске атомной кристаллической структуры ряда относительно простых соединений (оксидов и т.п.) $[8,10]$. В данной работе МПГА применен для вычислений на 8-ядерной ПК неизвестных кристаллических структур комплексных соединений платиновых металлов. Особенностью таких соединений служит наличие лигандов, представляющих, как правило, достаточно хорошо известные структурные фрагменты, которые могут использоваться в качестве первичных строительных единиц структуры (с последующим ее уточнением). Проблемой МПГА, как и других методов глобальной оптимизации в структурном анализе (также использующих структурные фрагменты), является настройка параметров поиска, от качества которой зависит вероятность сходимости структурных моделей к истинной структуре. В данной работе применена методика, использующая эмпирическую настройку параметров МПГА в процессе поиска известных кристаллических структур, близких по составу и сложности к исследуемым. В результате по МПГА найдены адекватные модели кристаллической структуры комплексных соединений $\left[\mathrm{Pd}\left(\mathrm{CH}_{3} \mathrm{NH}_{2}\right)_{4}\right]\left[\mathrm{PdBr}_{4}\right]$ и $\left[\mathrm{Pt}\left(\mathrm{NH}_{3}\right)_{5} \mathrm{Cl}\right] \mathrm{Br}_{3}$, которые хорошо уточняются методом полнопрофильного анализа DDM. B модели 1-й структуры атомы Pd располагаются в фиксированных частных позициях в ячейке, а по МПГА определены позиции $\mathrm{Br}$ и легких атомов $\mathrm{C}$ и $\mathrm{N}$ (в позициях которых затем уточнены по $\mathrm{DDM}$ группы $\mathrm{CH}_{3}$ и $\mathrm{NH}_{2}$ ). В модели 2-й структуры по МПГА определены позиции трех атомов $\mathrm{Br}$ и положение структурного фрагмента $\mathrm{Pt}\left(\mathrm{NH}_{3}\right)_{5} \mathrm{Cl}$ (вернее, $\mathrm{PtN}_{5} \mathrm{Cl}$, к которому далее могут быть добавлены атомы водорода). В вычислительном плане 2-я структурная модель является более сложной и определятся на сотом поколении эволюции, на порядок дольше, чем первая. Это связано с вычислительной сложностью одновременного определения позиции в ячейке и пространственной ориентации структурного фрагмента $\mathrm{PtN}_{5} \mathrm{Cl}$. Планируется дальнейшее развитие описанной методики структурного анализа с помощью МПГА при определении кристаллической структуры ряда других комплексных соединений платиновых металлов.

\section{Список литературы}

[1] Inorganic Crystal Structure Database. FIZ Karlsruhe. http://www.fiz-karlsruhe.de/icsd.html.

[2] Cambridge Structural Database. http://www.ccdc.cam.ac.uk/products/csd/

[3] Young R.A. The Rietveld Method, Oxford University Press, 1995, 298 p.

[4] Cerny R., Favre-Nicolin V. Direct space methods of structure determination from powder diffraction: principles, guidelines, perspectives, Z. Kristallogr, 2007, 222, 105-113.

[5] Meden A., Ivana Radosavljevic Evans. Structure determination from powder diffraction data: past, present and future challenges, Crystal Research and Technology, 2015, 1-12, DOI 10.1002/ crat. 201500048 . 
[6] Kenneth D.M. Harris. Fundamentals and applications of genetic algorithms for structure solution from powder X-ray diffraction data, Computational Materials Science, 2009, 45(1), 16-20.

[7] Якимов Я.И., Кирик С.Д., Семенкин Е.С. и др. Эволюционный метод моделирования кристаллической структуры вещества по данным порошковой дифракции. Журнал СФУ. Химия, 2013 6(2), 180-191 [Yakimov Ya.I., Kirik S.D., Semenkin E.S. at al. The Evolutionary Method of Modeling a Crystal Structure by Powder Diffraction Data, J. Sib. Fed. Univ. Chem., 2013 6(2), 180-191 (in Russian)]

[8] Залога А.Н., Бураков С.В., Семенкин Е.С., Якимов И.С. Мультипопуляционный генетический алгоритм моделирования кристаллической структуры вещества из рентгенодифракционных данных. Журнал СФУ. Химия, 2014 7(4), 573-581 [Zaloga A.N., Burakov S.V., Semenkin E.S., Yakimov I.S. Multipopulation Genetic Algorithm for Simulation of the Crystal Structure from X-Ray Diffraction Data, J. Sib. Fed. Univ. Chem.,573-581 (in Russian)]

[9] A. Le Bail. The profile of a Bragg reflection for extracting intensities. Powder Diffraction: Theory and Practice, ed. by R.E. Dinnebier \& S.J.L. Billinge, Royal Society of Chemistry, Cambridge, 2008, 134-165.

[10] Burakov S.V., Zaloga, A. N., Semenkin E.S., Yakimov I. S. Research on convergence of multipopulation binary- and real-coded genetic algorithms for solution of crystal structure from X-Ray powder diffraction data, Crystal Research and Technology, 2015, 50(9-10), 724-728.

[11] Solovyov L.A. Full-profile refinement by derivative difference minimization, J. Appl. Cryst., 2004, 37, 743-749.

[12] Favre-Nicolin V., Cerny R. FOX, free objects for crystallography: a modular approach to ab initio structure determination from powder diffraction, J. Appl. Cryst., 2002, 35, 734-743.

[13] Синтез комплексных соединений металлов платиновой группьл. Справочник. Отв. ред. И.И. Черняев. М.: Наука, 1964, с. 160-161 [Synthesis of complex compounds of platinum group metals. Guide. Ed. by I.I.Cherniaev. Moscow, Nauka,1964, p. 160-161 (in Russian)]

[14] Altomare A., Caliandro R., Camalli M., Cuocci C., Giacovazzo C., A. G. G. Moliterni and R. Rizzi, J. Appl. Crystallogr., 2004, 37, 1025.

[15] CheckCIF. http://checkcif.iucr.org/

[16] Crystal Impact Diamond ver. 3.2k. Scientific Computing World, 2002, 63, 19-21. 